\title{
On the supersymmetric completion of $R+R^{2}$ gravity and cosmology
}

\author{
Sergio Ferrara, ${ }^{a, b, 1}$ Renata Kallosh $^{c}$ and Antoine Van Proeyen ${ }^{d}$ \\ a Physics Department, Theory Unit, CERN, \\ CH 1211, Geneva 23, Switzerland \\ ${ }^{b}$ INFN - Laboratori Nazionali di Frascati, \\ Via Enrico Fermi 40, I-00044 Frascati, Italy \\ ${ }^{c}$ Stanford Institute for Theoretical Physics and Department of Physics, Stanford University, \\ Stanford, CA 94305-4060, U.S.A. \\ ${ }^{d}$ Instituut voor Theoretische Fysica, KU Leuven, \\ Celestijnenlaan 200D, B-3001 Leuven, Belgium \\ E-mail: sergio.ferrara@cern.ch, kallosh@stanford.edu, \\ Antoine.VanProeyen@fys.kuleuven. be
}

ABSTRACT: We revisit and clarify the supersymmetric versions of $R+R^{2}$ gravity, in view of the renewed interest to these models in cosmology. We emphasize that the content of the dual standard supergravity theory in the old minimal formulation necessarily includes two massive chiral multiplets, that we call the inflaton and the goldstino. We point out that the presence of these multiplets is model independent in the old minimal formulation and therefore any theory that contains a single chiral multiplet fails to be a supersymmetric generalization of the $R+R^{2}$ gravity. The supergravity interactions of the two chiral multiplets are encoded in a superpotential mass term and an arbitrary Kähler potential for the goldstino multiplet. The implication for cosmology of the supersymmetric $R+R^{2}$ gravity is also discussed.

KEYwords: Supergravity Models, Cosmology of Theories beyond the SM

ARXiv EPRINT: 1309.4052

\footnotetext{
${ }^{1}$ On leave of absence from Department of Physics and Astronomy, University of California Los Angeles, CA 90095-1547 U.S.A.
} 


\section{Contents}

1 Introduction 1

$\begin{array}{lll}2 & R+R^{2} \text { supergravity } & 2\end{array}$

2.1 Manifestly superconformal action 2

2.2 The dual to $R+R^{2}$ supergravity 3

2.3 The dual of " $F(R)$ supergravity" revisited 4

3 The structure of " $\boldsymbol{F}(\boldsymbol{R})$ Supergravity" $\quad \mathbf{5}$

3.1 Review of " $F(R)$ Supergravity" and cosmology 5

3.2 The action in components 6

$\begin{array}{lll}4 & \text { Discussion } & 7\end{array}$

A Elements of conformal tensor calculus $\quad 9$

\section{Introduction}

We discuss higher curvature theories of supergravity which can be considered as the supersymmetric version of the $R+R^{2}$ bosonic theory. The supersymmetric extension depends on the off-shell formulations of the supergravity. For minimal formulation with six bosonic degrees of freedom for the auxiliary fields, the $R+R^{2}$ theory is dual to a standard (two derivative) supergravity. In the old minimal case the linearized analysis [1] reveals two massive chiral multiplets, with the same mass, one of which contains the scalaron, i.e. the gravitational degree of freedom of the bosonic theory. We will call the first chiral multiplet $\Phi$ an "inflaton multiplet" and the second chiral multiplet $S$, a "goldstino multiplet", which will be explained in the context of inflationary evolution.

The full non-linear theory of the $R+R^{2}$ and generalizations thereof in old minimal supergravity was found in [2] and was shown to reduce to a standard two-derivative supergravity of two chiral superfields. In the new minimal supergravity full non-linear theory of the $R+R^{2}$ was found in [3] and was shown to be given by a standard two-derivative supergravity interacting with a massive vector multiplet. However, while the new minimal extension appears to be unique some freedom exists in the old minimal one. This freedom manifests in an arbitrary Kähler function for the goldstino chiral multiplet whose $\theta=0$ component is the sgoldstino. The name of this second multiplet comes from the observation that during inflation the auxiliary field $F_{S}=\frac{\partial W}{\partial S}$ is not vanishing and is responsible for supersymmetry breaking during inflation. In absence of this Kähler function one of the two chiral multiplets is no longer dynamical and the theory is not any longer the supersymmetrization of the $R+R^{2}$ gravity. 
The paper is organized as follows. In section 2 we review $R+R^{2}$ supergravity and its dual standard two-derivative supergravity description as considered in [2]. In the same section we revisit what in the literature was called " $F(R)$ supergravity" $[4,5]$. This is the situation where only one chiral multiplet is dynamical. In this case the supergravity interaction of this chiral multiplet has a fixed Kähler potential but an arbitrary superpotential. In section 3 we present the component expression of " $F(R)$ supergravity". The resulting action shows that while the vector auxiliary field $A_{\mu}$ appears algebraically and can be eliminated using its algebraic equations of motion, the auxiliary field $X$ appears covered by a derivative and is therefore propagating. This completely agrees with the linearized analysis of [1]. Note that to confine the analysis to a sector where $X$ only appears algebraically, gives an inconsistent result, as already noticed in [6]. Our analysis provides a detailed explanation why " $F(R)$ supergravity" $[4,5]$ has only terms linear in bosonic curvature $R$, which is important for the cosmological applications. Section 4 provides a discussion of our findings. In an appendix some elements of a conformal tensor calculus useful for understanding our results in components are given.

\section{$2 \quad R+R^{2}$ supergravity}

\subsection{Manifestly superconformal action}

In the old minimal supergravity the most general $R+R^{2}$ theory is given by a Lagrangian of the form [2]

$$
\mathcal{L}=-\left[S_{0} \cdot \bar{S}_{0}\right]_{D}+\left[S_{0} \cdot \bar{S}_{0} \cdot h\left(\frac{\mathcal{R}}{S_{0}}, \frac{\overline{\mathcal{R}}}{\bar{S}_{0}}\right)\right]_{D}+\left[S_{0}^{3} \cdot W\left(\frac{\mathcal{R}}{S_{0}}\right)\right]_{F} .
$$

This action has a manifest superconformal symmetry, and we write it using notations of superconformal calculus, reviewed in [7]. Old minimal supergravity uses a chiral compensating multiplet $S_{0}$ of Weyl weight 1 . Another chiral multiplet, a curvature multiplet $\mathcal{R}$ of weight 1 , is defined as [8]

$$
\mathcal{R}=\left(S_{0}\right)^{-1} \cdot \Sigma\left(S_{0}\right)=\left(S_{0}\right)^{-1} \cdot T\left(\bar{S}_{0}\right),
$$

where the operation $\Sigma$ or $T$ is defined in (A.1). The multiplication of chiral multiplets leads to new chiral multiplets, and $[\ldots]_{F}$ denotes the ' $F$ ' action formula on a chiral multiplet of weight 3 . On the other hand, $[\ldots]_{D}$ indicate the $D$ action formula on a real multiplet of weight 2. We use the symbol · for products to distinguish the use of curly brackets as arguments of a function from a multiplication by an expression in brackets.

The function $h$ is a real function, and $W$ is holomorphic. In order to have an $R+R^{2}$ supergravity it is essential that $h_{R \bar{R}} \equiv \frac{\partial^{2} h}{\partial \mathcal{R} \partial \mathcal{R}} \neq 0$. In fact, in absence of this term, as we will show later, the theory widely considered in the literature $[4,5]$ is no longer a supersymmetric extension of the $R+R^{2}$ theory. In particular, it does not contain two chiral multiplets, as required by a linearized analysis of [1]. In fact, when $h_{R \bar{R}}=0$ the goldstino multiplet is no longer dynamical. 


\subsection{The dual to $R+R^{2}$ supergravity}

The dual theory is obtained by introducing two chiral superfields, $S$ and $\Phi$, both of weight 0 , so that (2.1) can be written as

$$
\mathcal{L}=-\left[S_{0} \cdot \bar{S}_{0}\right]_{D}+\left[S_{0} \cdot \bar{S}_{0} \cdot h(S, \bar{S})\right]_{D}+\left[S_{0}^{3} \cdot\left(W(S)-3 \Phi \cdot\left(\frac{\mathcal{R}}{S_{0}}-S\right)\right)\right]_{F} .
$$

We also write the superpotential term $W$ as follows

$$
W(S)=S \cdot g(S)+\lambda,
$$

where $\lambda$ is a constant. By using the lemma, eq. (11) of [2], which is also explained in (A.2),

$$
\left[\Phi \cdot \mathcal{R} \cdot S_{0}^{2}\right]_{F}=\left[S_{0} \cdot \bar{S}_{0} \cdot(\Phi+\bar{\Phi})\right]_{D}+\text { tot.der }
$$

we obtain

$$
\mathcal{L}=\left[S_{0} \cdot \bar{S}_{0} \cdot(-1-3 \Phi-3 \bar{\Phi}+h(S, \bar{S}))\right]_{D}+\left[S_{0}^{3}(S \cdot(g(S)+3 \Phi)+\lambda)\right]_{F} .
$$

This defines the Kähler potential and superpotential (see e.g. (17.67) in [7]) as

$$
K(\Phi, S, \bar{\Phi}, \bar{S})=-3 \log \left(\frac{1}{3}+\Phi+\bar{\Phi}-\frac{1}{3} h(S, \bar{S})\right), \quad W(\Phi, S)=S \cdot(g(S)+3 \Phi)+\lambda .
$$

Now we observe that the chiral function $g(S)$ can be removed by defining

$$
\Phi^{\prime}=\Phi+\frac{1}{3} g(S), \quad h^{\prime}(S, \bar{S})=h(S, \bar{S})+g(S)+\bar{g}(\bar{S}) .
$$

We finally obtain, according to [2]

$$
\mathcal{L}_{\text {dual }}=\left[S_{0} \cdot \bar{S}_{0} \cdot\left(-1-3 \Phi^{\prime}-3 \bar{\Phi}^{\prime}+h^{\prime}(S, \bar{S})\right)\right]_{D}+\left[S_{0}^{3} \cdot\left(\lambda+3 S \cdot \Phi^{\prime}\right)\right]_{F} .
$$

We notice that the Kähler potential in (2.9) is of the no-scale type [9, 10], while the superpotential is not, because of the $S \cdot \Phi^{\prime}$ term. In fact, the no-scale model would give rise to a massless mode, which is not present in the action (2.9).

A model with no-scale Kähler potential, and the superpotential as in (2.9) with $\lambda=0$ has been constructed in [11]. It reproduces the bosonic $R+R^{2}$ model without unstable directions. In the $R+R^{2}$ supergravity theory, the corresponding expression of the $h$ function in $(2.1)$ is

$$
S_{0} \cdot \bar{S}_{0} \cdot h\left(\frac{\mathcal{R}}{S_{0}}, \overline{\overline{\mathcal{R}}} \bar{S}_{0}\right)=S_{0} \cdot \bar{S}_{0}+3 \mathcal{R} \cdot \overline{\mathcal{R}}-\zeta \cdot \frac{(\mathcal{R} \cdot \overline{\mathcal{R}})^{2}}{S_{0} \cdot \bar{S}_{0}}, \quad W\left(\frac{\mathcal{R}}{S_{0}}\right)=-3 \frac{\mathcal{R}}{S_{0}}
$$

The corresponding Kähler potential and superpotential of the dual theory are [11]

$$
K=-3 \log \left(\Phi+\bar{\Phi}-S \cdot \bar{S}+\frac{1}{3} \zeta \cdot(S \cdot \bar{S})^{2}\right), \quad W=3 S \cdot(\Phi-1),
$$

where $\Phi$ is the inflaton multiplet and $S$ is a goldstino multiplet. More general models in this class corresponding to an action (2.1) can have extra sgoldstino-dependent terms: 
in the superpotential one can add $W(S)$ and in the Kähler potential one can add some additional terms in $h(S, \bar{S})$. If these more general terms do not destroy the property of the model that $S=0$ is a minimum of the potential, these more general models will have the same cosmological observables as the ones in [11]. This adds additional features to the cosmological attractors described in [12]. Modified non-scale models resembling the Starobinsky model have also been investigated $[13,14]$.

The equivalence between (2.6) and (2.9) can also be understood by writing in (2.1)

$$
W\left(\frac{\mathcal{R}}{S_{0}}\right)=\frac{\mathcal{R}}{S_{0}} \cdot g\left(\frac{\mathcal{R}}{S_{0}}\right)+\lambda
$$

and using once more the lemma (A.2) we obtain

$$
\left[\frac{\mathcal{R}}{S_{0}} \cdot g\left(\frac{\mathcal{R}}{S_{0}}\right) \cdot S_{0}^{3}\right]_{F}=\left[\left[g\left(\frac{\mathcal{R}}{S_{0}}\right)+\bar{g}\left(\frac{\overline{\mathcal{R}}}{\bar{S}_{0}}\right)\right] \cdot S_{0} \cdot \bar{S}_{0}\right]_{D}
$$

which can then be absorbed in a redefinition as in (2.8). This shows that the $F(\mathcal{R})=$ $\mathcal{R} \cdot g(\mathcal{R})$ term is completely irrelevant when a non-trivial $h$-function with $h_{S \bar{S}} \neq 0$ is introduced.

\subsection{The dual of " $F(R)$ supergravity" revisited}

We can now retrieve as a particular case of the dual theory the " $F(R)$ supergravity" $[4,5]$ when $h_{S \bar{S}}=0$. In this case $h^{\prime}=g(S)+\bar{g}(\bar{S})$ so that $(2.9)$ becomes

$$
\mathcal{L}_{\text {dual }}=\left[S_{0} \cdot \bar{S}_{0} \cdot\left(-1-3 \Phi^{\prime}-3 \bar{\Phi}^{\prime}+g(S)+\bar{g}(\bar{S})\right)\right]_{D}+\left[S_{0}^{3} \cdot\left(\lambda+3 S \cdot \Phi^{\prime}\right)\right]_{F} \cdot
$$

By defining $\Phi=\Phi^{\prime}-\frac{1}{3} g(S)$ we get

$$
\mathcal{L}_{\text {dual }}=\left[S_{0} \cdot \bar{S}_{0} \cdot(-1-3 \Phi-3 \bar{\Phi})\right]_{D}+\left[S_{0}^{3} \cdot(\lambda+S \cdot(3 \Phi+g(S))]_{F} .\right.
$$

Since $S$ is not dynamical, we can now integrate over $S$ so we obtain the superpotential term $\lambda+w(\Phi)$ given by the Legendre transform

$$
w(\Phi)=-\left.S^{2} \cdot g^{\prime}(S)\right|_{(S \cdot g(S))^{\prime}=-3 \Phi} .
$$

The final Lagrangian is

$$
\mathcal{L}_{\text {dual }}=\left[S_{0} \cdot \bar{S}_{0} \cdot(-1-3 \Phi-3 \bar{\Phi})\right]_{D}+\left[S_{0}^{3} \cdot(\lambda+w(\Phi))\right]_{F}
$$

The Lagrangian (2.17) is dual to " $F(R)$ supergravity" [4,5], where only the $X$ auxiliary field becomes propagating and not the $R+R^{2}$ scalaron mode and not the $\partial_{\mu} A^{\mu}$ auxiliary scalar, see section 3 . In this situation only the vector field $A_{\mu}$ is auxiliary and satisfies an algebraic equation. 


\section{The structure of " $F(R)$ Supergravity"}

\subsection{Review of " $F(R)$ Supergravity" and cosmology}

The theories that have been called " $F(R)$ Supergravity" in $[4,5]$ are defined in superspace as

$$
\int \mathrm{d}^{4} x \mathrm{~d}^{2} \theta \mathcal{E} \cdot F(\mathcal{R}(x, \theta))+\text { h.c. },
$$

where $\mathcal{E}$ is the chiral superspace density, and $\mathcal{R}(x, \theta)$ is a chiral superfield containing the spacetime curvature $R(x)$ in the $\theta^{2}$ part:

$$
\mathcal{R}(x, \theta)=X+\ldots+\theta^{2} \cdot R(x)+\ldots .
$$

This action does not contain the bosonic terms $R+\alpha R^{2}$. Since in $N=1$ supersymmetry $\theta^{2 n}=0$ for $n>1$, integration of $F(\mathcal{R})$ over $\mathrm{d}^{2} \theta$ does not produce a non-linear dependence on space-time curvature $R(x) .{ }^{1}$ However, if the first component $X$ in (3.2) would be an auxiliary field, its elimination could produce $R^{2}$ or higher powers.

For example, with the choice made by Ketov and Starobinsky [5]

$$
F(\mathcal{R})=\frac{1}{2} f_{1} \mathcal{R}+\frac{1}{2} f_{2} \mathcal{R}^{2}+\frac{1}{6} f_{3} \mathcal{R}^{3},
$$

the supersymmetric action $(3.1)$ according to $[4,5]$ has the bosonic part

$$
\mathcal{L}_{\text {bos }}=\sqrt{g} \cdot \operatorname{Re} F^{\prime}(\bar{X}) \cdot\left(\frac{2}{3} R+8 X \cdot \bar{X}\right)+6 \sqrt{g} \cdot \operatorname{Re}(X \cdot F(\bar{X})),
$$

where $X$ is the complex auxiliary scalar of the supergravity multiplet. Since the equation of motion for the auxiliary field $X$ following from (3.4) is algebraic, one solves $X(x)$ in terms of $R(x)$ and in approximation of high curvature one produces the non-linear in $R(x)$ action of the form

$$
\mathcal{L}=\frac{1}{2} \sqrt{g} \cdot\left(R+\frac{R^{2}}{6 M^{2}}+\frac{3 \sqrt{105}}{100} \frac{R^{3 / 2}}{m}\right),
$$

where $M, m$ depend on $f_{i}$ in (3.3). Such actions have been used by cosmologists, see for example [15] for applications, such as reheating and non-Gaussianity in supergravity $R^{2}$-inflation.

However, we will show in section 3.2 that the action (3.5) is unrelated to the supersymmetric expression in (3.1), because the field $X$ appears with derivatives. The supersymmetric action (3.1) in fact has some extra terms beyond the ones in (3.4) depending on $A_{\mu}$, an auxiliary field of supergravity. Rescaling ${ }^{2}$ the result of section 3.2 reads

$$
\begin{aligned}
\mathcal{L}_{\text {bos }}= & \sqrt{g} \cdot \operatorname{Re} F^{\prime}(\bar{X}) \cdot\left(\frac{2}{3} R+8 X \cdot \bar{X}\right)-12 \sqrt{g} \cdot \operatorname{Re}(X \cdot F(\bar{X})) \\
& +4 \sqrt{g} \cdot \operatorname{Re} F^{\prime}(\bar{X}) \cdot A^{\mu} \cdot A_{\mu}-4 \sqrt{g} \cdot A^{\mu} \cdot \partial_{\mu} \operatorname{Im} F^{\prime}(\bar{X})
\end{aligned}
$$

\footnotetext{
${ }^{1}$ For example we may look at the $\mathcal{R}^{2}$ term in $F(\mathcal{R}(x, \theta))$. The bosonic term $R^{2}(x)$ there comes with $\theta^{4}$, which vanishes for $N=1$ supersymmetry since there are only 2 components $\theta_{\alpha}$.

${ }^{2}$ The variable $X$ of section 3.2 is replaced here by $X / \sqrt{3}$ and $F$ by $4 F /(3 \sqrt{3})$.
} 
If $F^{\prime \prime}(\bar{X})=0$ one finds that $A_{\mu}=0$, however for $F^{\prime \prime}(\bar{X}) \neq 0$ one finds that upon elimination of $A^{\mu}$ on its equations of motion, there are extra terms in the bosonic action. For example, in case of (3.3) the action includes terms proportional to (see complete expression below)

$$
f_{2} \partial_{\mu} X \cdot \partial^{\mu} \bar{X}+\ldots
$$

Therefore the complete bosonic part of the supersymmetric action (3.1) does not lead to an algebraic equation of motion for $X$, this field is not auxiliary anymore, it is propagating, and in fact is the only propagating complex scalar degree of freedom.

\subsection{The action in components}

We use the superconformal methods for $N=1$ supergravity with a chiral compensating multiplet $S_{0}$, which is reviewed in [7]. The multiplet is identified by its first component, and we use therefore the same name $S_{0}$ for this complex scalar with Weyl weight 1 . Using this compensating multiplet implies that in usual supergravity (without higher curvature terms) we have as auxiliary fields: the vector field of the Weyl multiplet, $A_{a}$, and the complex scalar that is the highest component of $S_{0}$. That scalar (in fact its complex conjugate) is proportional to the lowest component of the kinetic multiplet $\Sigma\left(S_{0}\right)$ (see appendix A), or thus also proportional to the lowest component of $\mathcal{R}$ defined in (2.2). The proportionality is always with factors of $S_{0}$, which in a gauge fixing as in (16.40) in [7], ${ }^{3}$ implies a proportionality with powers of the gravitational coupling constant $\kappa$. We will parametrize it as the lowest component of the chiral multiplet that appears in (2.1):

$$
\bar{X}=\frac{\mathcal{R}}{S_{0}}=\frac{\Sigma\left(S_{0}\right)}{S_{0}^{2}} .
$$

$\bar{X}$ has Weyl weight 0 . We will thus construct

$$
\mathcal{L}=\left(-\frac{1}{2}\right)\left[S_{0}^{3} \cdot F(\bar{X})\right]_{F},
$$

which is, up to normalization, the same as (3.1), or as $(2.1)$ with $W(\bar{X})=\frac{1}{2}[\bar{X}-F(\bar{X})]$ and $h=0$. In order to evaluate (3.9), we need the highest components of the various multiplets. Using the definition (3.8) and (16.36), which gives the highest component of $\Sigma(Z)$ for any chiral multiplet $Z$, the various multiplets have the following highest components:

$$
S_{0} \rightarrow S_{0}^{2} \cdot X, \quad \Sigma\left(S_{0}\right) \rightarrow \square^{C} \bar{S}_{0}, \quad \bar{X} \rightarrow S_{0}^{-2} \cdot \square^{C} \bar{S}_{0}-2 S_{0} \cdot X \cdot \bar{X} .
$$

Therefore, the bosonic part of (3.9) is

$$
\mathcal{L}_{\text {bos }}=-\frac{1}{2} \sqrt{g} \cdot\left\{3 S_{0}^{4} \cdot X \cdot F(\bar{X})+S_{0}^{3} \cdot F^{\prime}(\bar{X}) \cdot\left(S_{0}^{-2} \cdot \square^{C} \bar{S}_{0}-2 S_{0} \cdot X \cdot \bar{X}\right)\right\}+\text { h.c. }
$$

We will now use a gauge-fixing of the dilatations, where $S_{0}$ is a constant. Then the superconformal d'Alembertian gets the simple form as in (16.42) We can use here (16.37) for the $\square^{C} \bar{S}_{0}$ :

$$
\square^{C} \bar{S}_{0}=\bar{S}_{0} \cdot\left[\mathrm{i} e^{a \mu} \cdot\left(\partial_{\mu} A_{a}+\omega_{\mu a b} \cdot A^{b}\right)+2 f_{\mu}{ }^{\mu}-A^{a} \cdot A_{a}\right]+\text { fermionic },
$$

\footnotetext{
${ }^{3}$ Such high equation numbers below refer always to this book.
} 
and $f_{\mu}{ }^{\mu}=-\frac{1}{12} R$. With the gauge fixing $S_{0}=\bar{S}_{0}=\sqrt{3}$, we obtain

$$
\begin{aligned}
\mathcal{L}_{\text {bos }}= & -\frac{1}{2} \sqrt{g} \cdot\{27 X \cdot F(\bar{X}) \\
& \left.+3 F^{\prime}(\bar{X}) \cdot\left[\mathrm{i} e^{a \mu}\left(\partial_{\mu} A_{a}+\omega_{\mu a b} \cdot A^{b}\right)-\frac{1}{6} R-A^{a} \cdot A_{a}-6 X \cdot \bar{X}\right]\right\}+ \text { h.c. } .
\end{aligned}
$$

Note that for the case $W(\bar{X})=0$, i.e. $F(\bar{X})=\bar{X}$, the term with the derivative of $A_{a}$ is a total derivative, and this reproduces pure supergravity as in (16.44), now rewritten in terms of $X$. In that case also $X$ is auxiliary.

After adding a total derivative, we can write

$$
\begin{aligned}
\mathcal{L}_{\text {bos }}= & -\frac{1}{2} \sqrt{g} \cdot\left\{27 X \cdot F(\bar{X})-3 \mathrm{i} A^{\mu} \cdot \partial_{\mu} F^{\prime}(\bar{X})\right. \\
& \left.+F^{\prime}(\bar{X}) \cdot\left[-\frac{1}{2} R-3 A^{\mu} \cdot A_{\mu}-18 X \cdot \bar{X}\right]\right\}+ \text { h.c. }
\end{aligned}
$$

The field equation that follows from (3.14) determines

$$
A_{\mu}=\frac{\partial_{\mu} \operatorname{Im}\left(F^{\prime}(\bar{X})\right)}{2 \operatorname{Re}\left(F^{\prime}(\bar{X})\right)} .
$$

The action after inserting this value is

$$
\begin{aligned}
\mathcal{L}_{\text {bos }}= & \sqrt{g} \cdot\left\{-27 \operatorname{Re}(X \cdot F(\bar{X}))+\frac{1}{2}\left(\operatorname{Re} F^{\prime}(\bar{X})\right) \cdot(R+36 X \cdot \bar{X})\right. \\
& \left.-\frac{3}{4} \frac{\left[\partial_{\mu} \operatorname{Im}\left(F^{\prime}(\bar{X})\right)\right]^{2}}{\operatorname{Re}\left(F^{\prime}(\bar{X})\right)}\right\} .
\end{aligned}
$$

Hence, the implications of $F(\bar{X})$ are as follows: we have seen above that a linear term in $\bar{X}$ (with minus sign for the signature of the gravity term) produces pure supergravity. Adding a constant term produces a cosmological term (anti-de Sitter). When $F^{\prime \prime}(\bar{X}) \neq 0$, there are two real scalar physical fields: the imaginary part of $F^{\prime}(\bar{X})$ has its kinetic term explicitly in the last term. The real part of $F^{\prime}(\bar{X})$ is coupled to $R$. The action in (3.16) is presented in a Jordan frame, and the transformation to Einstein frame transforms this term as

$$
\mathcal{L}_{\mathrm{J}}=\frac{1}{2} \sqrt{g} \cdot \operatorname{Re} F^{\prime}(\bar{X}) \cdot R \rightarrow \mathcal{L}_{\mathrm{E}}=\sqrt{g} \cdot\left[\frac{1}{2} R-\frac{3}{4}\left(\frac{\partial_{\mu} \operatorname{Re} F^{\prime}(\bar{X})}{\operatorname{Re} F^{\prime}(\bar{X})}\right)^{2}\right] .
$$

So this theory describes a propagating complex scalar $(X=S+\mathrm{i} P)$ and is not an $R+R^{2}$ theory.

\section{Discussion}

In this paper we have revisited the supersymmetric completion of $R+R^{2}$ supergravity in the old minimal formulation. The manifestly superconformal action is given in (2.1). It is defined by a real function $h\left(\frac{\mathcal{R}}{S_{0}}, \frac{\overline{\mathcal{R}}}{S_{0}}\right)$ and a holomorphic function $W\left(\frac{\overline{\mathcal{R}}}{\bar{S}_{0}}\right)$. Here $S_{0}$ is a chiral conformon supermultiplet and $\mathcal{R}$ is the curvature supermultiplet. 
We have elucidated its degrees of freedom and the corresponding properties of the dual theory, in agreement with the original results of [2]. We stress the fact that any supersymmetric theory of $R+R^{2}$ gravity has to contain two chiral multiplets with some universal interactions. The first multiplet, is the inflaton $\Phi$, the second one is the goldstino $S$, according to their role during inflation.

We have found a new form of the Kähler-type symmetry between $h\left(\frac{\mathcal{R}}{S_{0}}, \frac{\overline{\mathcal{R}}}{S_{0}}\right)$ and $W\left(\frac{\overline{\mathcal{R}}}{S_{0}}\right)$ at the level of the superconformal theory, before the extra symmetries absent in Poincaré supergravity are fixed. Namely, we have shown that there is a possibility to absorb the part of the superpotential of the form $W(S)=S \cdot g(S)$ into the modification of the real function $h(S, \bar{S}) \rightarrow h(S, \bar{S})+g(S)+\bar{g}(\bar{S})$.

We described the relation of this class of theories with the cosmological model of inflation in [11], where the corresponding Kähler potential and superpotential of the ordinary two-derivative supergravity are given in (2.11). During inflation only the real scalar of the inflaton multiplet (the inflaton) is evolving; the 3 other scalars are stabilized in this model. The most general models dual to a supersymmetric completion of $R+R^{2}$ in (2.1) may add to [11] some arbitrary superpotentials $W(S)$, depending only on the goldstino multiplet, as well as more general goldstino depending terms $A(S, \bar{S})$ in the Kähler potential of the form $K_{A}=-3 \log \left(\Phi+\bar{\Phi}-S \cdot \bar{S}+\frac{1}{3} \zeta \cdot(S \cdot \bar{S})^{2}+A(S, \bar{S})\right)$. If in presence of these new terms $W(S)$ and $A(S, \bar{S})$ the generalized models still have a stable minimum at the vanishing sgoldstino, we find a new attractor property of these models in addition to the one described in [12]: the inflaton potential and, consequently, the cosmological observables during inflation do not depend on the choice of $W(S)$ and $A(S, \bar{S})$. An example of such a deformation is a superpotential $g S^{3}$ with a sufficiently small $g$.

We have then revisited the so-called " $F(R)$ supergravity" [4, 5], where only one chiral multiplet occurs. We clarified the physical degrees of freedom of the gravity theory and its dual counterpart and showed that the bosonic action of " $F(R)$ supergravity" [4, 5] is linear in the space-time curvature $R(x)$ and does not have non-linear terms in the curvature. In particular, it does not contain $R^{2}(x)$ term required for inflation in this model.

It is interesting to observe that in the cosmological models based on supersymmetry of which $R+R^{2}$ supergravity is a particular realization, three chiral multiplets come to play a role: in addition to an inflaton multiplet $\Phi$ and the goldstino multiplet $S$, also the conformon (the conformal compensator multiplet $S_{0}$ ) has an important role. The later helps in the use of some hidden symmetries that are remnants of the full underlying superconformal formulation of the theory.

\section{Acknowledgments}

We are grateful to S. Cecotti, A. Linde, and M. Porrati for stimulating discussions.

R.K. is supported by Stanford Institute for Theoretical Physics (SITP), the NSF Grant No. 0756174 and the John Templeton foundation grant 'Quantum Gravity Frontiers'. S.F. is supported by ERC Advanced Investigator Grant n. 226455 Supersymmetry, Quantum Gravity and Gauge Fields (Superfields). A.V.P. is supported in part by the FWO Vlaanderen, Project No. G.0651.11, and in part by the Interuniversity Attraction Poles 
Programme initiated by the Belgian Science Policy (P7/37). S.F and A.V.P. are grateful to SITP for the hospitality at Stanford where this work was finalized.

\section{A Elements of conformal tensor calculus}

The chiral projection is in superspace an operation $\bar{D}^{2}$, applied to the complex conjugate superfield. We define on a chiral multiplet $S$ with (Weyl, chiral) weight (see table 17.1) $(w, c)=(1,1)$ the map to another chiral multiplet by

$$
\Sigma(S)=T(\bar{S}): S(w, c)=(1,1) \rightarrow \Sigma(S)=T(\bar{S})(w, c)=(2,2) .
$$

This is the map that is explicitly given in (16.36), which associates to the chiral multiplet $S=\left(X, P_{L} \Omega, F\right)$ the multiplet starting with $\bar{F}$.

For any two chiral multiplets $\Lambda$ (with $w=0$ ) and $Z$ (with $w=1$ ) we have the lemma

$$
[Z \cdot \bar{Z} \cdot(\Lambda+\bar{\Lambda})]_{D}=[\Lambda \cdot Z \cdot \Sigma(Z)]_{F} .
$$

We show this by checking the bosonic parts. The left-hand side is a function of chiral multiplets, and we can use (17.19):

$$
\begin{aligned}
{[Z \cdot \bar{Z} \cdot(\Lambda+\bar{\Lambda})]_{D}=\sqrt{g} \cdot(} & -\mathcal{D}_{\mu}(\Lambda Z) \cdot \mathcal{D}^{\mu} \bar{Z}+\Lambda_{F} \cdot Z \cdot \bar{F}+\Lambda \cdot F \cdot \bar{F} \\
& \left.\quad-\frac{1}{6} \Lambda \cdot Z \cdot \bar{Z} \cdot R(\omega)\right)+ \text { h.c. } \\
= & \sqrt{g} \cdot\left((\Lambda \cdot Z) \cdot \square^{C} \bar{Z}+\Lambda_{F} \cdot Z \cdot \bar{F}+\Lambda \cdot F \cdot \bar{F}\right)+\text { h.c. }
\end{aligned}
$$

where $\Lambda_{F}$ is the $F$ component of $\Lambda$. From (16.36) we see that $\square^{C} \bar{Z}$ is the $F$ component of $\Sigma(Z)$. Thus the three terms are the 3 ways in which to get the $F$ component indicated in the right-hand side of (A.2), which proves the lemma.

Open Access. This article is distributed under the terms of the Creative Commons Attribution License which permits any use, distribution and reproduction in any medium, provided the original author(s) and source are credited.

\section{References}

[1] S. Ferrara, M.T. Grisaru and P. van Nieuwenhuizen, Poincaré and Conformal Supergravity Models With Closed Algebras, Nucl. Phys. B 138 (1978) 430 [InSPIRE].

[2] S. Cecotti, Higher derivative supergravity is equivalent to standard supergravity coupled to matter. 1., Phys. Lett. B 190 (1987) 86 [INSPIRE].

[3] S. Cecotti, S. Ferrara, M. Porrati and S. Sabharwal, New minimal higher derivative supergravity coupled to matter, Nucl. Phys. B 306 (1988) 160 [INSPIRE].

[4] S. Ketov, $F(R)$ supergravity, AIP Conf. Proc. 1241 (2010) 613 [arXiv:0910.1165] [INSPIRE].

[5] S.V. Ketov and A.A. Starobinsky, Embedding $\left(R+R^{2}\right)$-Inflation into Supergravity, Phys. Rev. D 83 (2011) 063512 [arXiv:1011.0240] [INSPIRE]. 
[6] S.J. Gates Jr. and S.V. Ketov, Superstring-inspired supergravity as the universal source of inflation and quintessence, Phys. Lett. B 674 (2009) 59 [arXiv:0901.2467] [INSPIRE].

[7] D.Z. Freedman and A. Van Proeyen, Supergravity, Cambridge University Press, Cambridge U.K. (2012).

[8] T. Kugo and S. Uehara, $N=1$ Superconformal Tensor Calculus: Multiplets With External Lorentz Indices and Spinor Derivative Operators, Prog. Theor. Phys. 73 (1985) 235 [INSPIRE].

[9] E. Cremmer, S. Ferrara, C. Kounnas and D.V. Nanopoulos, Naturally Vanishing Cosmological Constant in $N=1$ Supergravity, Phys. Lett. B 133 (1983) 61 [InSPIRE].

[10] J.R. Ellis, A. Lahanas, D.V. Nanopoulos and K. Tamvakis, No-Scale Supersymmetric Standard Model, Phys. Lett. B 134 (1984) 429 [inSPIRE].

[11] R. Kallosh and A. Linde, Superconformal generalizations of the Starobinsky model, JCAP 06 (2013) 028 [arXiv: 1306.3214] [INSPIRE].

[12] R. Kallosh and A. Linde, Universality Class in Conformal Inflation, JCAP 07 (2013) 002 [arXiv:1306.5220] [INSPIRE].

[13] J. Ellis, D.V. Nanopoulos and K.A. Olive, Starobinsky-like Inflationary Models as Avatars of No-Scale Supergravity, JCAP 10 (2013) 009 [arXiv:1307.3537] [INSPIRE].

[14] J. Ellis, D.V. Nanopoulos and K.A. Olive, No-Scale Supergravity Realization of the Starobinsky Model of Inflation, Phys. Rev. Lett. 111 (2013) 111301 [arXiv:1305.1247] [INSPIRE].

[15] Y. Watanabe and J. Yokoyama, Gravitational modulated reheating and non-Gaussianity in Supergravity $R^{2}$ inflation, Phys. Rev. D 87 (2013) 103524 [arXiv: 1303.5191] [INSPIRE]. 